\title{
Effect of Telbivudine Versus Other Nucleos(t)ide Analogs on HBeAg Seroconversion and Other Outcomes in Patients with Chronic Hepatitis B: A Network Meta-Analysis
}

\author{
Xieer Liang · Rong Fan · Jian Sun · Javed Shaikh · Ankush Taneja • \\ Subhajit Gupta · Kamal Hamed (D) \\ To view enhanced content go to www.advancesintherapy.com \\ Received: January 14, 2016 / Published online: February 26, 2016 \\ (c) The Author(s) 2016. This article is published with open access at Springerlink.com
}

\section{ABSTRACT}

Introduction: A comprehensive and up-to-date network meta-analysis (NMA) helps to determine the comparative efficacies of nucleos(t)ide analogs (NAs) in patients with chronic hepatitis B (CHB). The aim of this NMA was to assess the efficacy of telbivudine versus adefovir, entecavir, lamivudine, and tenofovir in nucleos(t)ide-naïve hepatitis $\mathrm{B}$ e antigen (HBeAg)-positive patients with CHB.

Methods: A systematic review was conducted to search Medline, Medline-In Process, EMBASE, and the Cochrane Central Register of Controlled Trials databases for publications of

Electronic supplementary material The online version of this article (doi:10.1007/s12325-016-0305-x) contains supplementary material, which is available to authorized users.

X. Liang $\cdot$ R. Fan $\cdot$ J. Sun

Hepatology Unit, Nanfang Hospital, Southern

Medical University, Guangzhou, China

J. Shaikh · A. Taneja · S. Gupta

Novartis Healthcare Pvt. Ltd., Hyderabad, India

K. Hamed ( $\square)$

Novartis Pharmaceuticals Corporation,

East Hanover, NJ, USA

e-mail: kamal.hamed@novartis.com randomized controlled trials (RCTs). NMA was performed to compare the efficacy outcomes of telbivudine versus other approved NAs at 1- and 2-year time points.

Results: A total of 75 RCTs were included in the systematic review. At the 1-year time point, telbivudine was associated with significantly higher rates of: (1) HBeAg seroconversion than adefovir [odds ratio (OR) 1.99 (95\% credible interval (CrI): 1.05, 3.45)], entecavir [OR 2.00 (95\% CrI: 1.44, 2.82)] and lamivudine [OR 1.49 (95\% CrI: 1.10, 2.03)]; (2) HBeAg loss than entecavir [OR 1.85 (95\% CrI: 1.28, 2.76)] and lamivudine [OR 1.62 (95\% CrI: 1.20, 2.24)]; (3) alanine aminotransferase (ALT) normalization than lamivudine [OR 1.50 (95\% CrI: 1.05, 2.21)]; and (4) hepatitis B virus (HBV) DNA suppression than adefovir [OR 2.77 (95\% CrI: 1.28, 5.45)] and lamivudine [OR 2.97 (95\% CrI: $1.99,4.53)]$. At the 2-year time point, the relative efficacy outcomes were not statistically significant.

Conclusion: At 1 year, telbivudine was superior to adefovir, entecavir and lamivudine in HBeAg seroconversion, and to entecavir and lamivudine in HBeAg loss. Telbivudine was also superior to lamivudine in ALT 
normalization and to adefovir and lamivudine in suppressing HBV DNA levels.

Funding: Novartis Pharma AG.

Keywords: Alanine aminotransferase; Chronic hepatitis B; Cirrhosis; Hepatitis B e antigen seroconversion; Infectious diseases; Meta-analysis; Nucleos(t)ide analogs

\section{INTRODUCTION}

Hepatitis B virus (HBV) infection is a major global public health problem. It is estimated that 240 million people are chronically infected with HBV worldwide and approximately 780000 deaths each year are attributed to hepatitis B [1]. Chronic hepatitis B (CHB) can cause severe liver inflammation and fibrosis, ultimately resulting in more serious complications such as cirrhosis, hepatic decompensation and hepatocellular carcinoma (HCC) [2]. In patients with $\mathrm{CHB}$, the presence of hepatitis $\mathrm{B}$ e antigen (HBeAg) indicates active HBV replication and more severe infection. Therefore, it may be useful to monitor HBeAg levels in patients to determine treatment response, as HBeAg seroconversion in HBeAg-positive patients with sustained undetectable HBV DNA may be considered as a potential end point in the treatment of CHB [3].

Currently approved therapies for $\mathrm{CHB}$ include two immune-based interferons [interferon- $\alpha$ (IFN- $\alpha)$ and pegylated IFN $\alpha$ (PEG-IFN $\alpha$-2a or $\alpha$-2b)] and five antiviral nucleos(t)ide analogs (NAs), namely adefovir, entecavir, lamivudine, telbivudine, and tenofovir. These antivirals do not completely eradicate $\mathrm{HBV}$, and hence the efficacy of these therapies is still unsatisfactory $[2,4]$. At present, NAs are most commonly used in HBeAg-positive patients with CHB. HBeAg seroconversion in patients with $\mathrm{CHB}$ is associated with favorable long-term outcomes, such as disease remission, lower incidence of cirrhosis and HCC, and higher survival rates [3, $5,6]$. A number of systematic literature reviews (SLRs) and meta-analyses have been published on the efficacy of NAs for the treatment of $\mathrm{CHB}$ [4, 7-10]. Although these meta-analyses evaluated the efficacy of NAs, their scope was limited primarily to direct comparisons of NAs without considering indirect evidence using a common comparator between two NAs, which were not compared against each other in a randomized controlled trial (RCT). There is only one published meta-analysis which used a mixed-treatment comparison of NAs [8], but that study evaluated only 1-year efficacy outcomes. It is necessary to compare the available NAs through direct and indirect comparisons beyond the 1-year time period to have a more comprehensive overview of the efficacy of current treatment options in CHB. This can help physicians with selecting the most appropriate NA treatment options based on comparative efficacies. A network meta-analysis (NMA) helps to synthesize and analyze data by comparing multiple treatments both directly and indirectly $[11,12]$. To address this need, we have performed SLR and NMA to compare the efficacy of the approved NAs. The aim of this meta-analysis was to assess the efficacy of telbivudine compared to adefovir, entecavir, lamivudine, and tenofovir in nucleos(t)ide-naïve HBeAg-positive patients with CHB.

\section{METHODS}

This SLR followed standard systematic review methodology endorsed by the Cochrane Collaboration [13] and the National Institute 
for Health and Care Excellence (NICE) in the UK [14]. The SLR was conducted and reported in accordance with the Preferred Reporting Items for Systematic Reviews and Meta-Analyses (PRISMA) guidelines [15]. This article is based on previously conducted studies and does not involve any new studies of human or animal subjects performed by any of the authors.

\section{Inclusion and Exclusion Criteria}

All RCTs with HBeAg-positive, nucleos(t)ide-naïve patients with $\mathrm{CHB}$ were identified. RCTs reporting both HBeAg-positive and -negative patients were considered if subgroup data for $\mathrm{HBeAg}$-positive patients were reported. Only those RCTs with interventions or comparators (adefovir, entecavir, lamivudine, telbivudine, tenofovir, and placebo), with reported outcomes on HBeAg seroconversion, HBeAg loss, HBV DNA levels, alanine aminotransferase (ALT) normalization, and hepatitis B surface antigen (HBsAg) loss and seroconversion, were eligible for inclusion. Exclusion criteria comprised animal and in vitro studies; studies of patients who were co-infected with hepatitis $C$, hepatitis $\mathrm{D}$, or human immunodeficiency viruses; studies of patients with decompensated liver disease; studies of HBeAg-negative patients; studies which did not report interventions, comparators, or the outcomes of interest; and studies which were published in languages other than English or Chinese. Review articles, editorials, case reports, case series, economic evaluations, abstracts, and poster presentations were also excluded.

\section{Search Strategy}

A comprehensive literature search of Medline, Medline-In Process, EMBASE, and the Cochrane
Central Register of Controlled Trials databases was performed for relevant published studies within the period from January 2004 to February 2015. The search strategy was based on the combination of medical subject headings (MeSH) and key words including "adefovir", "entecavir", "lamivudine", "telbivudine", "tenofovir", "chronic hepatitis B", "hepatitis B", "randomized controlled trial", "random allocation", "clinical trial", "double-blind method", and "single-blind method". The majority of relevant published studies were in English and Chinese, and thus both English and Chinese publications were included in the literature search.

\section{First-Level Screening of Citations}

All the studies retrieved from the literature search were screened based on the title and abstract supplied with each citation. The inclusion/exclusion criteria were uniformly applied across all the studies. Two independent reviewers for English and two for Chinese publications screened the retrieved abstracts, and any discrepancies between reviewers were reconciled by a third independent reviewer. Studies that did not meet the eligibility criteria were excluded, and reasons for exclusion were documented. Full-text copies of all references that met the inclusion criteria were then downloaded.

\section{Second-Level Screening of Citations}

The inclusion/exclusion criteria were uniformly applied across all the full-text publications. The same two reviewers for English and two for Chinese publications screened all the manuscripts, and any discrepancies between reviewers were reconciled by a third independent reviewer. Studies that did not 
meet the eligibility criteria were excluded, and reasons for exclusion were documented. Studies that met the inclusion criteria were subjected to data extraction.

\section{Data Extraction}

The same reviewers extracted data independently in a data extraction template, with any discrepancies resolved by a third independent reviewer. Data were extracted based on different information from a study, such as objectives, methods, inclusion and exclusion criteria, baseline demographic and clinical characteristics, interventions, efficacy outcomes, and conclusion. Studies with multiple publications were linked to one another and extracted as a single study. The full texts of the relevant articles were examined to determine the relevance of data on study objectives, design, inclusion/exclusion criteria, patients, interventions, and efficacy outcomes. The RCTs that met the inclusion criteria for the review were critically appraised for quality based on the recommendations by NICE [14].

\section{Assessments of End Points}

The primary end point of the analysis was HBeAg seroconversion in patients with $\mathrm{CHB}$. HBeAg seroconversion was defined as HBeAg loss and the appearance of anti-HBe antibodies. Secondary end points included rates of HBeAg loss, normalization of ALT, undetectable HBV DNA levels, and HBsAg loss and seroconversion. HBV DNA levels less than 1000 copies/mL (HBV DNA $<200,300,400,500$ copies $/ \mathrm{mL}$, etc.) reported in studies were pooled together and analyzed for the undetectable HBV DNA end point. The study end points were analyzed at 1-year (48-52 weeks) and 2-year (96-104 weeks) time points.

\section{Statistical Analysis}

The meta-analysis was performed employing statistical methods that combine data from various studies to obtain a coherent picture of treatment outcomes and compare various treatment options. The statistical models related the underlying outcome to the effect of treatments and any other factors (covariates).

The models for conducting NMA were taken from the Report of the ISPOR Task Force on Indirect Treatment Comparisons Good Research Practices Part 2 and NICE TSD2 [16, 17]. For this analysis, Bayesian models were used. To assess the heterogeneity with respect to study location, age, and baseline HBV DNA levels, exploratory analyses and random effects Bayesian models with study level covariates were used.

The statistical software $\mathrm{R}$ (version $\geq 3.0 .2$, $\mathrm{R}$ Foundation for Statistical Computing, Vienna, Austria) was used for data pre-processing and statistical analysis. The integrated GUI for R, Rstudio (version $\geq 0.97 .551$ ), was used to run all the $\mathrm{R}$ scripts. The package R2WinBUGS was used to retrieve WinBUGS 1.4 from $\mathrm{R}$ in order to run the Bayesian Markov Chain Monte Carlo algorithm. The results were reported in terms of odds ratios with corresponding 95\% credible intervals.

\section{RESULTS}

\section{Search Results}

The results of the literature search are summarized in Fig. 1. The search yielded 5499 publications. Of these, 1719 studies were duplicates due to the overlap of records across the databases. All 3780 studies went through first-level screening (title/abstract screening). 

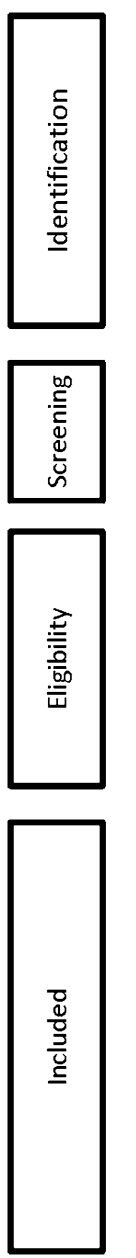

Records identified through OVID

database search

$\mathrm{N}=5499$

Cochrane CENTRAL, $N=869$

Embase, $\mathrm{N}=3032$

Medline (including In-Process), N = 1598

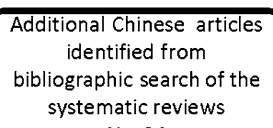

Fig. 1 Flow diagram of the literature search

Following this, 432 publications were found to be eligible. These were subjected to second-level screening (full-text screening) and there were 86 publications remaining. The number of publications excluded at each level of screening and the corresponding reasons for exclusion are presented in Fig. 1. A total of 24 Chinese publications were additionally identified through bibliographic search (three of these were duplicate Chinese articles), resulting in a total of 107 publications. As some publications were linked to one another, the final list of publications included 75 studies (37 English publications and 38 Chinese publications).

\section{Study Characteristics}

Of the 75 included studies, 29 (39\%) English and 32 (43\%) Chinese language studies were active controlled. Only nine (12\%) studies in total were placebo controlled. Of the remaining five studies, one compared lamivudine with untreated controls and four were dose-ranging studies. Of the 38 Chinese language studies, 29 (76\%) were single center, whereas of the 37 English language studies, 26 (70\%) were multi-center. Almost all of the Chinese language studies were phase IV trials and 14 (38\%) of the English language studies were phase III, with only four studies reported to be 
phase II. More than half of the included studies (52\%) had 1-year duration in the randomized phase.

In the included RCTs, lamivudine was the most commonly assessed comparator accounting for 24 studies. This was followed by placebo, which was the comparator in 12 of the included studies. NAs were assessed as monotherapy in 58 of the included studies. Overall, $19 \%$ of the included studies may be at risk of bias: of these, $15 \%$ due to blinding methodology and 4\% due to statistical analysis. When compared to English language studies, a larger number of Chinese language studies did not report the method of randomization, allocation concealment, blinding, or statistical analysis.

Randomization and allocation concealment were reported to be adequate in $24 \%$ of the included studies. Apart from blinding, more than $50 \%$ of the included studies reported a low risk of bias in terms of baseline characteristics, patient withdrawals, selective outcome reporting, and statistical analysis. About 15\% of the included English language studies were considered to be at high risk of bias because of their open-label design.

All the studies were conducted in HBeAg-positive CHB patients. The details of the demographic and disease characteristics of study participants at baseline are listed in Table S1 in the online supplementary material. The sample size of the included studies ranged from 14 to 921 [18, 19]. The median age of the patients ranged from 24 to 44 years $[10,20]$. The majority of studies recruited primarily male patients; $37 \%$ of these studies reported a male population greater than 70\%. The studies reported ALT levels above $100 \mathrm{IU} / \mathrm{L}$. Of 11 studies which reported information on HBV genotypes, more than $50 \%$ of the recruited patients had genotype $\mathrm{C}$ as the major viral genotype, indicating that the majority of the study population represented is of Southeast Asian descent [21]. The proportion of patients with genotype B ranged from $8 \%$ to $41 \%$ among the included studies which reported information on HBV genotype $[22,23]$. Table $\mathrm{S} 2$ in the online supplementary material shows the total numbers of patients in each treatment regimen and the corresponding numbers of patients with reported $\mathrm{HBeAg}$ seroconversion, $\mathrm{HBeAg}$ loss, ALT normalization, and undetectable HBV DNA.

\section{HBeAg Seroconversion}

Figure 2 shows the full network diagram for evidence of treatment regimens with $\mathrm{HBeAg}$ seroconversion outcomes at the 1-year time point. A total of 40 studies reported HBeAg seroconversion results. The relative efficacy of NAs at the 1-year time point demonstrated that telbivudine was superior to adefovir, entecavir, and lamivudine (Fig. 3a). The relative efficacy outcomes of telbivudine versus other NAs at the 2-year time point were not statistically significant. There were a relatively small number of studies (14 studies) which reported outcomes at the 2-year time point.

\section{HBeAg Loss}

Thirty studies reported HBeAg loss results. The NMA on relative efficacy at the 1-year time point showed that telbivudine was superior to entecavir and lamivudine for HBeAg loss in patients with $\mathrm{CHB}$ (Fig. 3b). The relative efficacy of NAs at the 2-year time point yielded no statistically significant results. 


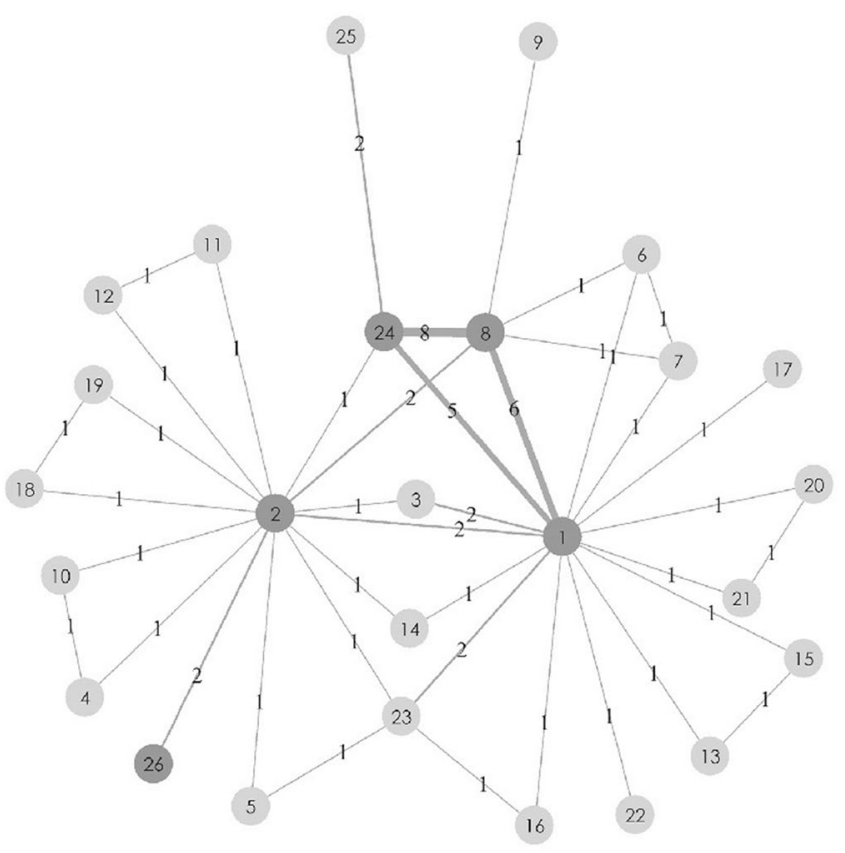

Fig. $2 \mathrm{HBeAg}$ seroconversion at 1 year-network diagram. The numbers $1,2,3$, etc. denote individual treatment regimens with corresponding full names given on the right side panel. This network diagram provides a summary of direct and indirect evidence from trials in a graphical manner. Each treatment is represented by a node, and a serial number is provided for each node representing the treatment regimen on the right side panel. The line

\section{ALT Normalization}

Thirty-two studies reported ALT normalization results. The relative efficacy of NAs at the 1-year time point is presented in Fig. 3c. The NMA demonstrated that telbivudine was superior to lamivudine in ALT normalization.

\section{Undetectable HBV DNA}

There were 34 studies that reported rates of undetectable HBV DNA at 1 year of treatment. The relative efficacy of NAs at the 1-year time point (Fig. 3d) demonstrated that telbivudine was superior to adefovir and lamivudine in suppressing HBV DNA levels. Tenofovir was

\begin{tabular}{|c|c|}
\hline Serial number & Treatment regimen \\
\hline 1 & Lamivudine $100 \mathrm{mg} / \mathrm{d}$ \\
\hline 2 & Adefovir $10 \mathrm{mg} / \mathrm{d}$ \\
\hline 3 & Adefovir $10 \mathrm{mg} / \mathrm{d}+$ Lamivudine $100 \mathrm{mg} / \mathrm{d}$ \\
\hline 4 & Adefovir $10 \mathrm{mg} / \mathrm{d}+\mathrm{IFN}$-alfa $5 \mathrm{mlU}$ \\
\hline 5 & Adefovir $30 \mathrm{mg} / \mathrm{d}$ \\
\hline 6 & Entecavir $0.01 \mathrm{mg} / \mathrm{d}$ \\
\hline 7 & Entecavir $0.1 \mathrm{mg} / \mathrm{d}$ \\
\hline 8 & Entecavir $0.5 \mathrm{mg} / \mathrm{d}$ \\
\hline 9 & Entecavir $0.5 \mathrm{mg} / \mathrm{d}+$ Tenofovir $300 \mathrm{mg} / \mathrm{d}$ \\
\hline 10 & IFN alfa $5 \mathrm{mlU}$ \\
\hline 11 & IFN alfa- 2 b 3 mlU qod \\
\hline 12 & IFN alfa-2b $3 \mathrm{mlU}$ qod + Adefovir $10 \mathrm{mg} / \mathrm{d}$ \\
\hline 13 & IFN alfa- 2 b 5 mlU qod \\
\hline 14 & Lamivudine $100 \mathrm{mg} / \mathrm{d}+$ Adefovir $10 \mathrm{mg} / \mathrm{d}$ \\
\hline 15 & Lamivudine $100 \mathrm{mg} / \mathrm{d}+\mathrm{IFN}$ alfa-2b $5 \mathrm{mlU}$ qd sequentia \\
\hline 16 & Lamivudine $25 \mathrm{mg} / \mathrm{d}$ \\
\hline 17 & No treatment \\
\hline 18 & Peg IFN alfa-2a $135 \mathrm{mcg}$ qw \\
\hline 19 & Peg IFN alfa-2a $135 \mathrm{mcg} q \mathrm{q}+$ Adefovir $10 \mathrm{mg} / \mathrm{d}$ \\
\hline 20 & Peg IFN alfa-2a $180 \mathrm{mcg}$ qw \\
\hline 21 & Peg IFN alfa-2a $180 \mathrm{mcg}$ qw + Lamivudine $100 \mathrm{mg} / \mathrm{d}$ \\
\hline 22 & Peg IFN alfa-2b $1.5 \mathrm{mcg} / \mathrm{kg} \mathrm{qw}+$ Lamivudine $100 \mathrm{mg} / \mathrm{c}$ \\
\hline 23 & Placebo \\
\hline 24 & Telbivudine $600 \mathrm{mg} / \mathrm{d}$ \\
\hline 25 & Telbivudine $600 \mathrm{mg} / \mathrm{d}+$ Adefovir $10 \mathrm{mg} / \mathrm{d}$ \\
\hline 26 & Tenofovir $300 \mathrm{mg} / \mathrm{d}$ \\
\hline
\end{tabular}

connecting two nodes represents the direct evidence comparing the treatments. The numbers between the lines represent the number of studies available for that particular treatment comparison. The dark nodes indicate approved monotherapy regimens, whereas light ones indicate other therapeutic regimens from which indirect evidence is obtained

superior to telbivudine in suppressing $\mathrm{HBV}$ DNA levels.

\section{Analysis of Heterogeneity}

None of the factors including study location, age, and baseline HBV DNA was found to affect the results.

\section{DISCUSSION}

This comprehensive and up-to-date NMA analyzed both direct and indirect evidence for the comparative efficacies of NAs. To ensure as comprehensive an approach as possible, we incorporated both English and Chinese 
publications. We included indirect evidence from combination therapies (as shown in Fig. 2). As NMA provides pooled efficacy estimates, the small sample size of some studies did not affect the overall results.

The 1-year results from this NMA revealed significant differences in HBeAg seroconversion when comparing telbivudine and most of the approved oral NAs. Telbivudine demonstrated superior efficacy over adefovir, entecavir and lamivudine in HBeAg seroconversion. The clinical importance of $\mathrm{HBeAg}$ seroconversion was reported in treatment guidelines. It is considered as a potential treatment end point in HBeAg-positive patients with undetectable HBV DNA and persistently normal ALT levels [3, 24]. HBeAg seroconversion is associated with favorable long-term outcomes, including reduced risk of cirrhosis or HCC [3]. A long-term study in patients with $\mathrm{CHB}$ showed that during a median follow-up of approximately nine years after spontaneous HBeAg seroconversion, the majority (67\%) of patients had sustained remission [5]. Another study reported that the rate of fibrosis progression was lower in patients

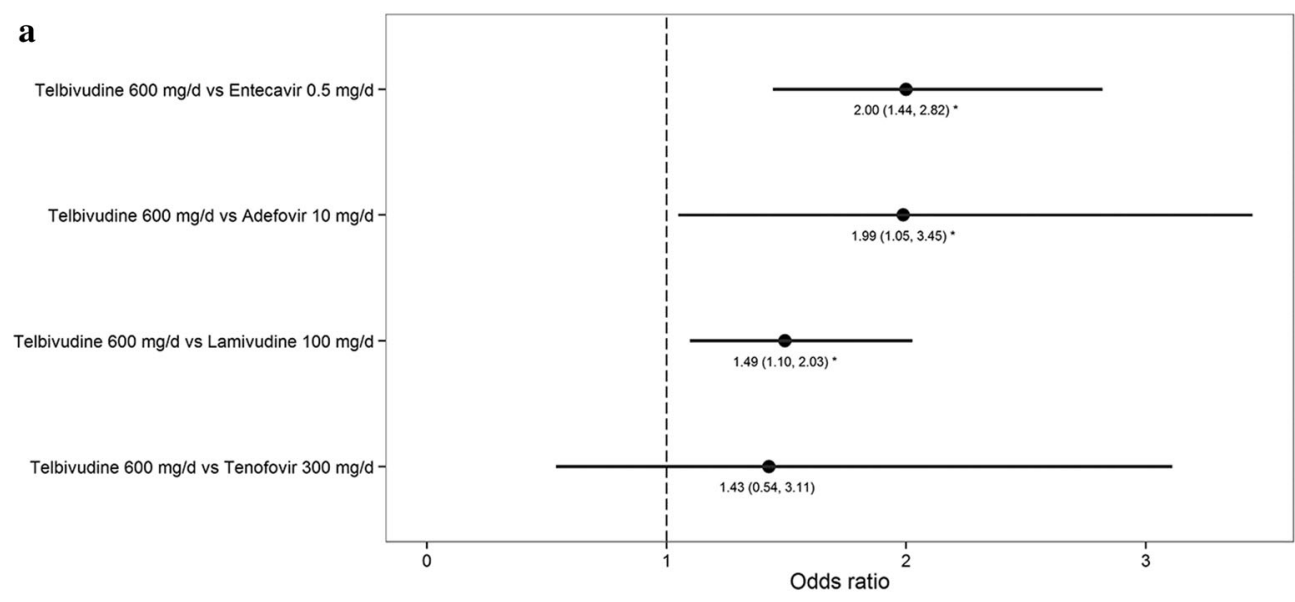

b

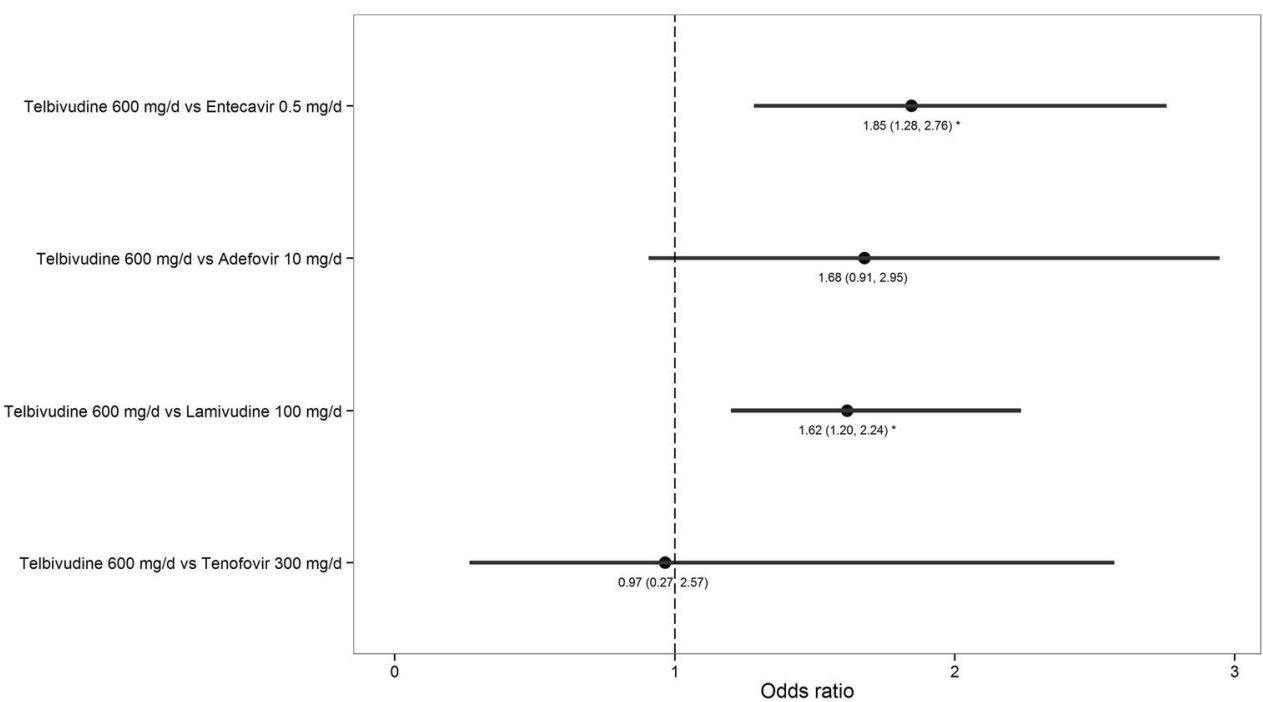

Fig. 3 Relative efficacy at 1 year (odds ratio, credible interval): a HBeAg seroconversion, b HBeAg loss, c ALT normalization, d undetectable HBV DNA 


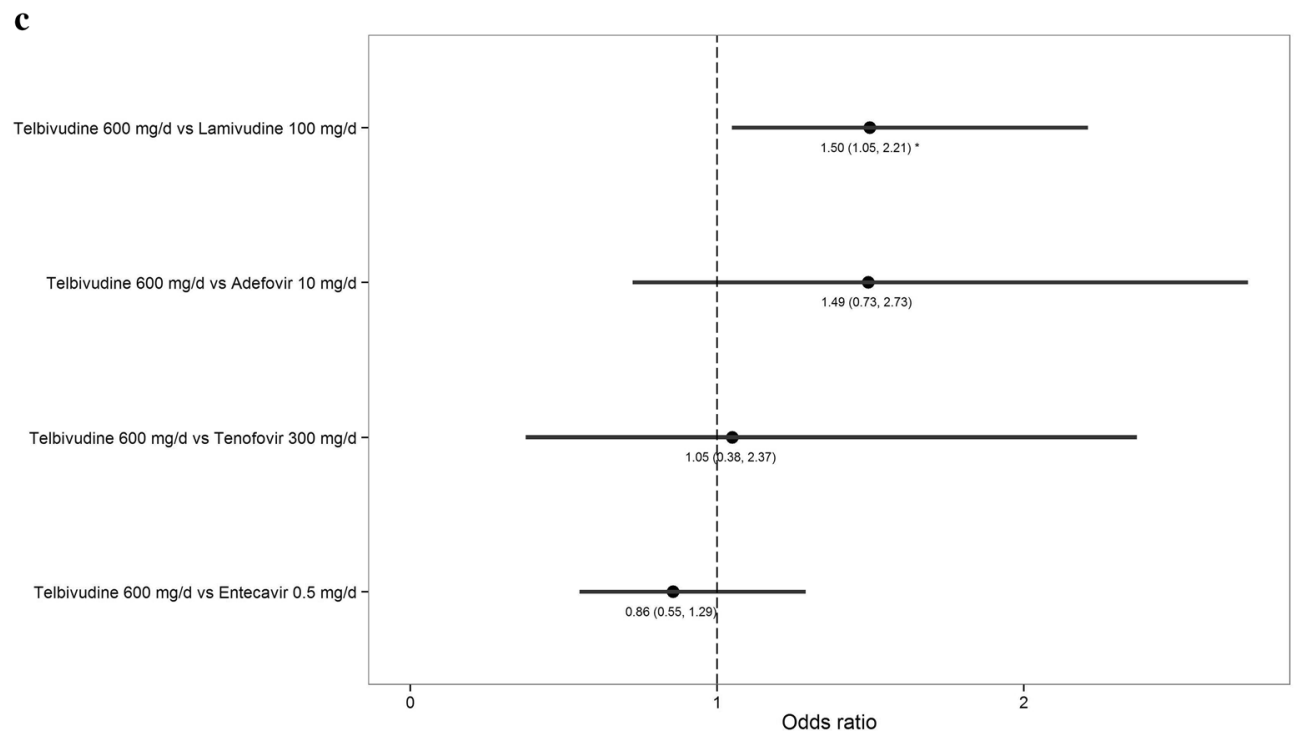

d

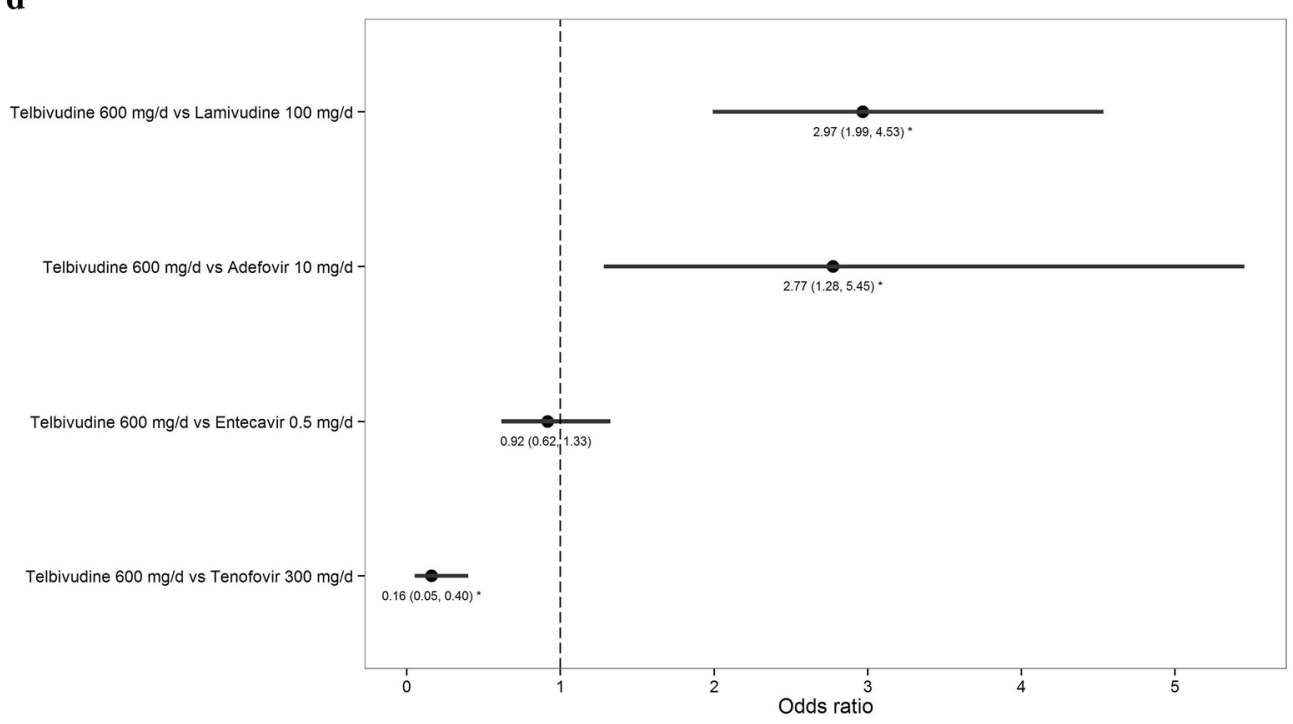

Fig. 3 continued

with sustained disease remission (HBeAg seroconversion and HBV DNA $<10^{4}$ copies $/ \mathrm{mL}$ at follow-up) as compared to patients who remained $\mathrm{HBeAg}$ positive [25]. These reports indicate that HBeAg seroconversion is an important end point in the treatment of patients with $\mathrm{CHB}$.

The present analysis also demonstrated that telbivudine was superior to entecavir and lamivudine for inducing HBeAg loss, and to lamivudine in ALT normalization. With regard to reducing $\mathrm{HBV}$ DNA levels, telbivudine demonstrated superior efficacy as compared to adefovir and lamivudine, and similar efficacy to entecavir. Outcomes at 1 year showed that tenofovir was superior to telbivudine in suppressing HBV DNA levels. However, there were only two studies with tenofovir which reported undetectable $\mathrm{HBV}$ DNA at the 1-year time point. Data from an earlier meta-analysis 
showed that compared to other NAs, tenofovir had the highest probability of achieving HBeAg seroconversion after 1 year of treatment. Tenofovir also showed significantly higher HBV DNA reduction than the other NAs. Entecavir was significantly superior to adefovir and lamivudine, whereas telbivudine showed superior efficacy than lamivudine [8]. However, this meta-analysis by Wiens et al. has two major limitations that were acknowledged by the authors. First, a random-effect meta-analysis software (ADDIS) was used, unlike the current analysis that was performed using WinBUGS 1.4 , a widely accepted software for conducting Bayesian NMA. Second, the previously published analysis included data from only nine RCTs of 48-52 weeks duration. Hence, results from this published analysis cannot be extrapolated or generalized to real-life situations.

Results from a prospective study showed that treatment intensification (Roadmap approach) with adefovir add-on therapy in patients with suboptimal virologic response (HBV DNA $\geq 300$ copies $/ \mathrm{mL}$ ) after 24 weeks of telbivudine treatment significantly improved efficacy outcomes at 2 years. This shows that adjustment of treatment strategy may be useful for patients with suboptimal virologic response to telbivudine treatment [23]. An earlier study with Roadmap approach in HBeAg-positive, nucleoside-naïve patients with CHB appeared to be an effective treatment approach. Telbivudine with conditional tenofovir intensification resulted in high rates of undetectable HBV DNA, ALT normalization, HBeAg/HBsAg clearance, and HBeAg seroconversion. There was neither virologic breakthrough nor resistance observed over 52 weeks of treatment [26]. Therefore, telbivudine and tenofovir add-on therapy, based on Roadmap approach, may be a useful strategy to optimize antiviral treatment outcomes in patients with suboptimal virologic response.

The superiority of telbivudine over entecavir with regard to HBeAg loss and HBeAg seroconversion in this 1-year NMA was in accordance with a previously published meta-analysis that directly compared telbivudine versus entecavir in treatment-naïve HBeAg-positive patients with CHB [10]. The published direct meta-analysis concluded that in nucleos(t)ide-naïve Asian patients with $\mathrm{CHB}$, assessed at 12, 24, and 48 weeks after starting the treatment, telbivudine was as effective as entecavir in HBV DNA suppression, but had higher rates of $\mathrm{HBeAg}$ loss and seroconversion as compared to entecavir [10]. In the long-term analysis at the 2-year time point, the relative efficacies of telbivudine versus other NAs with regard to $\mathrm{HBeAg}$ loss and $\mathrm{HBeAg}$ seroconversion were not statistically significant. The analysis was not conducted for other outcomes due to the limited number of studies reporting data at 2 years. Earlier, 2-year results from the GLOBE study (ClinicalTrials.gov identifier: NCT00057265) showed that a subgroup of HBeAg-positive patients with baseline HBV DNA $<9 \log _{10}$ copies/mL, ALT level $\geq 2 \times$ upper limit of normal, and undetectable HBV DNA at week 24 achieved high rates of undetectable HBV DNA (89\%) and HBeAg seroconversion $(52 \%)$ with low rate of telbivudine resistance $(1.8 \%)$ at 2 years. Therefore, the baseline characteristics of patients with $\mathrm{CHB}$ and undetectable serum HBV DNA at treatment week 24 (early virologic response) may be considered as the strongest predictors of long-term outcomes of telbivudine treatment [27].

Several studies have shown that high HBV DNA levels, high ALT levels, and HBeAg-positivity are independent risk factors 
for the development of HCC and cirrhosis [28, 29]. Thus, effective antiviral therapy and induction of $\mathrm{HBeAg}$ seroconversion may lower the risk of developing HCC. The possible mode of action for the telbivudine-mediated high rate of $\mathrm{HBeAg}$ seroconversion is through direct inhibition of viral replication and stimulation of the host immune response [30]. This suggests that telbivudine provides potential benefits for patients with HBeAg-positive CHB. It is important to note that newer-generation oral NAs need to be developed with the objective of inducing higher rates of $\mathrm{HBeAg}$ loss and seroconversion that are sustained over a long period of time after the end of treatment. This can provide the possibility of a finite therapy for HBeAg-positive patients with CHB [6].

There are some limitations to this analysis. It primarily evaluated $\mathrm{HBeAg}$ seroconversion and other efficacy data. However, viral resistance and adverse events due to NA treatment were not assessed, although these are key factors when selecting a particular therapy for CHB. An evaluation of the cost-effectiveness of NAs was not part of this NMA. The analysis mainly reported results from RCTs with 1-year of treatment. A limited number of studies reported outcomes at the 2-year time point; therefore, a more robust comparison was not feasible. Hence, the NMA results are not reliable at the 2-year time point due to the small number of studies. Furthermore, the long-term ( $\geq 5$ years) efficacies of NAs and the potential benefits in the reduction of liver-related complications were not determined. A previous review on the use of NAs in HBeAg-positive CHB patients reported that rates of $\mathrm{HBeAg}$ loss increased up to $50 \%$ and anti-HBe seroconversion up to $37 \%$ at year 6 of treatment [31]. Given the chronic nature of the disease, cohort studies to evaluate the long-term outcomes after treatment with different NAs would be important.

\section{CONCLUSIONS}

This SLR and NMA demonstrated that in nucleos(t)ide-naïve HBeAg-positive patients with $\mathrm{CHB}$, telbivudine was superior to adefovir, entecavir, and lamivudine in HBeAg seroconversion, and to entecavir and lamivudine in $\mathrm{HBeAg}$ loss at 1 year of treatment. Telbivudine also showed a superior response as compared to lamivudine in ALT normalization and to adefovir and lamivudine in suppressing HBV DNA levels.

\section{ACKNOWLEDGMENTS}

Article processing charges and the open access charge for this NMA were funded by Novartis Pharma AG. All named authors meet the International Committee of Medical Journal Editors (ICMJE) criteria for authorship of this manuscript, take responsibility for the integrity of the work as a whole, and have given final approval to the version to be published. The authors thank Rajeeb Ghosh, Novartis Healthcare Pvt. Ltd., for medical writing support. Funding for writing support was provided by Novartis Pharma AG.

Disclosures. Xieer Liang and Rong Fan have nothing to disclose. Jian Sun has served as a speaker for Novartis and BMS, and as an advisory board member for Gilead. Javed Shaikh, Ankush Taneja, and Subhajit Gupta are employees of Novartis Healthcare Pvt. Ltd. Kamal Hamed is an employee of Novartis Pharmaceuticals Corporation. 
Compliance with Ethics Guidelines. This article is based on previously conducted studies and does not involve any new studies of human or animal subjects performed by any of the authors.

Open Access. This article is distributed under the terms of the Creative Commons Attribution-NonCommercial 4.0 International License (http://creativecommons.org/licenses/ by-nc/4.0/), which permits any noncommercial use, distribution, and reproduction in any medium, provided you give appropriate credit to the original author(s) and the source, provide a link to the Creative Commons license, and indicate if changes were made.

\section{REFERENCES}

1. WHO fact sheet on Hepatitis B. March 2015 [cited 2015, 5 May]; http://www.who.int/mediacentre/ factsheets/fs204/en/.

2. Koumbi L. Current and future antiviral drug therapies of hepatitis B chronic infection. World J Hepatol. 2015;7:1030-40.

3. Guidelines for the prevention, care and treatment of persons with chronic hepatitis B infection. Geneva: World Health Organization; 2015 Mar. Publications of the World Health Organization are available on the WHO website (http://www.who. int).

4. Ke W, Liu L, Zhang C, et al. Comparison of efficacy and safety of tenofovir and entecavir in chronic hepatitis B virus infection: a systematic review and meta-analysis. PLoS One. 2014;9:e98865.

5. Hsu YS, Chien RN, Yeh CT, et al. Long-term outcome after spontaneous HBeAG seroconversion in patients with chronic hepatitis B. Hepatology. 2002;35:1522-7.

6. Liaw YF. HBeAg seroconversion as an important end point in the treatment of chronic hepatitis $B$. Hepatol Int. 2009;3:425-33.

7. Feng X, Yan L, Lu J, et al. Effects of nucleoside analogue on patients with chronic hepatitis b-associated liver failure: meta-analysis. PLoS One. 2013;8:e54773.

8. Wiens A, Lenzi L, Venson R, et al. Comparative efficacy of oral nucleoside or nucleotide analog monotherapy used in chronic hepatitis B: a mixed-treatment comparison meta-analysis. Pharmacotherapy. 2013;33:144-51.

9. Liang J, Jiang MJ, Deng X, Zhou XX. Efficacy and safety of telbivudine compared to entecavir among HBeAg+ chronic hepatitis B patients: a meta-analysis study. Hepat Mon. 2013;13:e7862.

10. Liu $H$, Wang $X$, Wan $G$, Yang $Z$, Zeng $H$. Telbivudine versus entecavir for nucleos(t)ide-naive HBeAg-positive chronic hepatitis B: a meta-analysis. Am J Med Sci. 2014;347:131-8.

11. Zhang Y, Kang S, Fang W, Wu X, Liang W. Network meta-analysis on prophylactic regimens against recurrent hepatitis B virus infection after liver transplantation. Hepatobiliary Surg Nutr. 2013;2:297-303.

12. Salanti G, Higgins JP, Ades AE, Ioaniddis JP. Evaluation of networks of randomized trials. Stat Methods Med Res. 2008;17:279-301.

13. Higgins JPT, Green S, editors. Cochrane handbook for systematic reviews of interventions version 5.1.0 [updated March 2011]. The Cochrane Collaboration, 2011. http://www.cochranehandbook.org.

14. National Institute for Health and Care Excellence. Guide to the methods of technology appraisal [Online]. http://www.nice.org.uk/article/pmg9/ chapter/foreword. Accessed 19 Feb 2015.

15. Moher D, Liberati A, Tetzlaff J, Altman DG, PRISMA Group. Preferred reporting items for systematic reviews and meta-analyses: the PRISMA statement. Ann Intern Med. 2009;151:264-9.

16. Hoaglin DC, Hawkins N, Jansen JP, et al. Conducting indirect-treatment-comparison and network-meta-analysis studies: report of the ISPOR Task Force on Indirect Treatment Comparisons Good Research Practices: part 2. Value Health. 2011;14:429-37.

17. Dias S, Sutton AJ, Ades AE, Welton NJ. Evidence synthesis for decision making 2: a generalized linear modeling framework for pairwise and network meta-analysis of randomized controlled trials. Med Decis Making. 2013;33:607-17.

18. Wong DK, Yuen MF, Ngai VW, Fung J, Lai CL. One-year entecavir or lamivudine therapy results in 
reduction of hepatitis B virus intrahepatic covalently closed circular DNA levels. Antiviral Ther. 2006;11:909-16.

19. Lai CL, Gane E, Liaw YF, et al. Telbivudine versus lamivudine in patients with chronic hepatitis B. N Engl J Med. 2007;357:2576-88.

20. Wang YD, Zhao CY, Wang $\mathrm{W}$, et al. Improved efficacy by individualized combination therapy with Peg IFN- $\alpha 2 \mathrm{a}$ and ADV in HBeAg positive chronic hepatitis B patients. Hepatogastroenterology. 2012;59:680-6.

21. Sunbul M. Hepatitis B virus genotypes: global distribution and clinical importance. World J Gastroenterol. 2014;20:5427-34.

22. Janssen HL, van Zonneveld $\mathrm{M}$, Senturk $\mathrm{H}$, et al. Pegylated interferon alfa- $2 \mathrm{~b}$ alone or in combination with lamivudine for HBeAg-positive chronic hepatitis B: a randomised trial. Lancet. 2005;365:123-9.

23. Sun J, Xie Q, Tan D, et al. The 104-week efficacy and safety of telbivudine-based optimization strategy in chronic hepatitis B patients: a randomized, controlled study. Hepatology. 2014;59:1283-92.

24. Sarin SK, Kumar M, Lau GK, et al. Asian-Pacific clinical practice guidelines on the management of hepatitis B: a 2015 update. Hepatol Int. 2016;10:1-98.

25. Hui CK, Leung N, Shek TW, et al. Sustained disease remission after spontaneous HBeAg seroconversion is associated with reduction in fibrosis progression in chronic hepatitis $B$ Chinese patients. Hepatology. 2007;46(3):690-8.

26. Piratvisuth TKP, Komolmit P, Tanwandee T, et al. 52 -week efficacy and safety of telbivudine with conditional tenofovir intensification at week 24 in HBeAg-positive chronic hepatitis B. PLoS One. 2013;8:e54279.

27. Zeuzem S, Gane E, Liaw YF, et al. Baseline characteristics and early on-treatment response predict the outcomes of 2 years of telbivudine treatment of chronic hepatitis B. J Hepatol. 2009;51:11-20.

28. Chen C, Yang HI, Su J, et al. Risk of hepatocellular carcinoma across a biological gradient of serum hepatitis B virus DNA level. JAMA. 2006;295:65-73.

29. Yang HI, Lu SN, Liaw YF, et al. Hepatitis B e antigen and the risk of hepatocellular carcinoma. N Engl J Med. 2002;347:168-74.

30. Evans A, Riva A, Cooksley H, et al. Programmed death 1 expression during antiviral treatment of chronic hepatitis B: impact of hepatitis B e-antigen seroconversion. Hepatology. 2008;48:759-69.

31. Buti M. HBeAg-positive chronic hepatitis B: why do I treat my patients with nucleos(t)ide analogs? Liver Int. 2014;34(Suppl 1):108-11. 